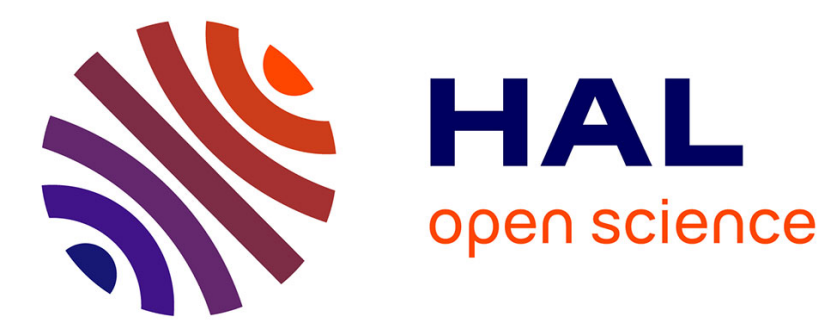

\title{
Effet de l'état structural d'une couche labourée sur sa rétention en eau
}

François Papy, Christophe Maitre, Philippe Lafleuriel, Eric Tesson

\section{To cite this version:}

François Papy, Christophe Maitre, Philippe Lafleuriel, Eric Tesson. Effet de l'état structural d'une couche labourée sur sa rétention en eau. Agronomie, 1986, 6 (6), pp.555-565. hal-00884910

\section{HAL Id: hal-00884910 https://hal.science/hal-00884910}

Submitted on 1 Jan 1986

HAL is a multi-disciplinary open access archive for the deposit and dissemination of scientific research documents, whether they are published or not. The documents may come from teaching and research institutions in France or abroad, or from public or private research centers.
L'archive ouverte pluridisciplinaire HAL, est destinée au dépôt et à la diffusion de documents scientifiques de niveau recherche, publiés ou non, émanant des établissements d'enseignement et de recherche français ou étrangers, des laboratoires publics ou privés. 


\title{
Effet de l'état structural d'une couche labourée sur sa rétention en eau
}

\author{
François PAPY \\ avec la collaboration de Christophe Maitre, Philippe Lafleuriel $\left({ }^{*}\right) \&$ Eric Tesson $\left({ }^{*}\right)$
}

I.N.R.A., Unité S.A.D., I.N.A. Paris-Grignon, F 78850 Thiverval-Grignon

(*) Etudiants à l'I.N.A. Paris-Grignon

RÉSUMÉ

\begin{abstract}
Une couche de terre labourée se caractérise par une forte variabilité spatiale de la structure avec la présence de discontinuités souvent subverticales. On peut tenir compte de cette hétérogénéité en distinguant plusieurs niveaux dans la description de l'état structural. L'étude vise à analyser comment chacun de ces niveaux influe sur la rétention en eau de la couche labourée. On représente l'état structural d'une couche labourée par une cartographie des unités homogènes à l'échelle du décimètre, repérées sur une section verticale d'un profil de sol. Chacune d'elles, appelées unités morphologiques, est caractérisée par l'état interne des éléments structuraux qui la constituent et par leur mode d'assemblage.

Au sein d'une même couche travaillée, on a mis en évidence des discontinuités de teneur pondérale en eau, liées aux discontinuités structurales, alors qu'il y a continuité dans les profils de charge établis par tensiométrie. Les différences de relation $\mathrm{pF}$-Humidité pondérale entre unités morphologiques, établies en laboratoire, expliquent les observations réalisées in situ. Dans la gamme des pF rencontrés au printemps dans un labour, les unités morphologiques dont l'état interne des éléments structuraux est compact, ont une teneur pondérale en eau plus faible que les autres.

En comparant le comportement hydrique de deux types de labour ( $\mathrm{O}:$ fragmenté ; $\mathrm{B}:$ en blocs avec vides), on est amené à émettre l'hypothèse d'une infiltration privilégiée de l'eau dans les interblocs du labour $B$, se traduisant par une moindre imbibition des blocs compacts. Ce dernier phénomène accentue les effets du premier. Ainsi, dans le comportement hydrique d'une couche labourée, différents phénomènes entrent en jeu aux différents niveaux utilisés pour décrire l'état structural.

Un des résultats de cette étude porte sur la façon de déterminer les jours disponibles.
\end{abstract}

Mots clés additionnels : Structure du sol, profil cultural, comportement hydrique, succion, infiltration.

An important feature of the ploughed layer of the soil is the spatial variability of its structure with the presence of subvertical discontinuities. This heterogeneity can be taken into account in distinguishing several levels in the description of the structural state. The objective of our survey was to analyse the influence of each of these levels on the water retention of the ploughed layer. The structure of the ploughed layer was represented by mapping the units which are homogeneous at the decimetre scale located on a vertical section of a profile. Each of these units, called "morphological units", could clearly be characterized by the internal state of its structural element sand by their mode of assembly. It was shown that, in a given ploughed layer, structural discontinuities exist in ponderal water content, whereas the tensiometer records indicate continuous hydraulic load profiles. The difference in the $\mathrm{pF}$ ponderal water content relation between morphological units, established in the laboratory, explained the observations made in situ. In the $\mathrm{pF}$ range encountered during spring ploughing, the structural elements with compact internal state had the lowest ponderal water content. Comparison of the hydric behaviour of two types of ploughed layers $(\mathrm{O}-$ fragmented ; $\mathrm{B}-$ with blocks separated by voids) showed that in $\mathrm{B}$ the gaps between blocks were preferential paths for the infiltration of water, resulting in lesser imbibition of compact blocks. This latter phenomenon increases the effects of the former one. Thus, to explain the hydric behaviour of a ploughed layer, different phenomena have to be involved at the different levels used for the structural state description. One result of this study concerns a way of determining work days for ploughing.

Additional key words : Soil structure, cultural profile, hydraulic behaviour, suction, infiltration. 


\section{INTRODUCTION}

Pour porter un jugement sur les systèmes de culture pratiqués et en proposer d'autres, l'agronome a besoin de comprendre le comportement du sol sous l'action des outils (MANICHON \& SEBILLOTTE, 1975 ; MANICHON, 1982a). En l'état actuel des connaissances, on ne sait proposer que des modèles de comportement qualitatifs à partir de la connaissance de deux critères : l'état hydrique et l'état structural (MANICHON, 1982b). Mais ces prévisions ne peuvent s'appliquer que juste avant l'intervention des outils. Pour les faire plus précocement, il faut être à la même de prévoir les comportements hydriques de la couche travaillée du sol sous l'action du climat.

Les transferts hydriques dans le sol ont fait l'objet de nombreuses études. Les modèles physiques les plus généraux mettent en jeu les phénomènes de transfert hydrique et de chaleur (BRUCKLER, travaux en cours). Ils ont, pour l'instant, été appliqués à des systèmes à structure plus simple qu'une couche travaillée dans laquelle on observe souvent une variation spatiale des caractéristiques structurales, marquée de discontinuités subverticales. Afin de permettre ultérieurement une modélisation du comportement de ce système complexe qu'est une couche travaillée (surtout juste après labour), nous avons cherché à relier ses propriétés de rétention d'eau à son état structural. Les principes élaborés par MANICHON (1982a), pour caractériser la morphologie d'un profil cultural ont été utilisés. Selon cet auteur, on peut rendre compte de l'hétérogénéité structurale de la couche travaillée, en distinguant plusieurs niveaux d'échelle.

A partir de l'observation d'une face verticale d'une fosse, suffisamment longue pour inclure la marque des derniers passages de tracteurs-outils (plusieurs mètres), on réalise une cartographie de la couche de terre à caractériser. Pour ce faire, on distingue des ensembles structuraux considérés comme homogènes à l'échelle centimétrique à décimétrique appelés unités morphologiques. Selon les cas, ces dernières apparaissent comme massives et continues, ou constituées d'un assemblage plus ou moins lâche d'éléments structuraux de plus petite taille.

L'état structural d'une couche travaillée est donc caractérisé à 3 niveaux (MANICHON, 1982b) :

- le niveau 1 des éléments structuraux dont l'état interne peut revêtir 2 modalités principales : $\Delta$ et $\Gamma$ (tabl. 1) ;

- le niveau 2 de l'assemblage d'éléments structuraux identiques constitutifs d'une unité morphologique dont on distingue 4 modalités (M, SD, SF, F) (tabl. 1) ;

- le niveau 3 de la disposition spatiale des différentes unités morphologiques au sein de la couche travaillée (tabl. 1, fig. 1).

L'analyse de l'effet de l'état structural d'une couche travaillée sur son comportement hydrique peut donc se formuler ainsi : Comment chacun des niveaux intervient-il sur la rétention en eau de l'ensemble du système (PAPY, 1984) ?

\section{MATÉRIEL ET MÉTHODES}

Nous avons réalisé sur le Centre expérimental de la Station d'Agronomie de Grignon (Yvelines), au cours de l'hiver 1983, des conditions de labour suffisamment différenciées dans un sol limono-argileux (tabl. 2) pour obtenir, en sortie d'hiver, 2 modalités de couche labourée :

- un type $O$ fragmenté,

- un type B en gros blocs compacts avec vides.

TABLEAU 1

Symboles utilisés pour caractériser l'état structural du sol à différents niveaux d'échelle (d'après MANicHON, 1982). Symbols used to characterize structural states at different levels (after MANICHON, 1982).

\begin{tabular}{|c|c|c|}
\hline Niveau & Echelle & Modalités retenues \\
\hline 1 & \multirow{2}{*}{ 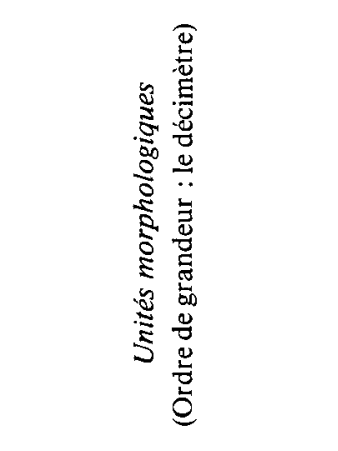 } & 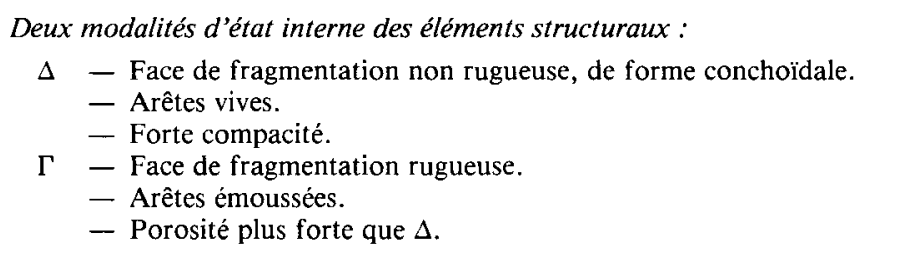 \\
\hline 2 & & $\begin{array}{l}\text { Quatre modalités de modes d'assemblage des éléments structuraux : } \\
\text { M - Massif (un seul élément structural). } \\
\text { SD - Eléments soudés difficilement discernables. } \\
\text { SF - Eléments soudés facilement discernables. } \\
\text { F - Eléments individualisés ; assemblage fragmentaire. }\end{array}$ \\
\hline 3 & 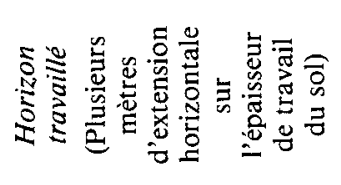 & $\begin{array}{l}\text { Deux modalités de distribution et d'organisation spatiale des unités mor- } \\
\text { phologiques: } \\
\text { B - Couche constituée de blocs denses et de vides centimétriques } \\
\text { o intercalaires. } \\
\text { - Couche fragmentée. }\end{array}$ \\
\hline
\end{tabular}




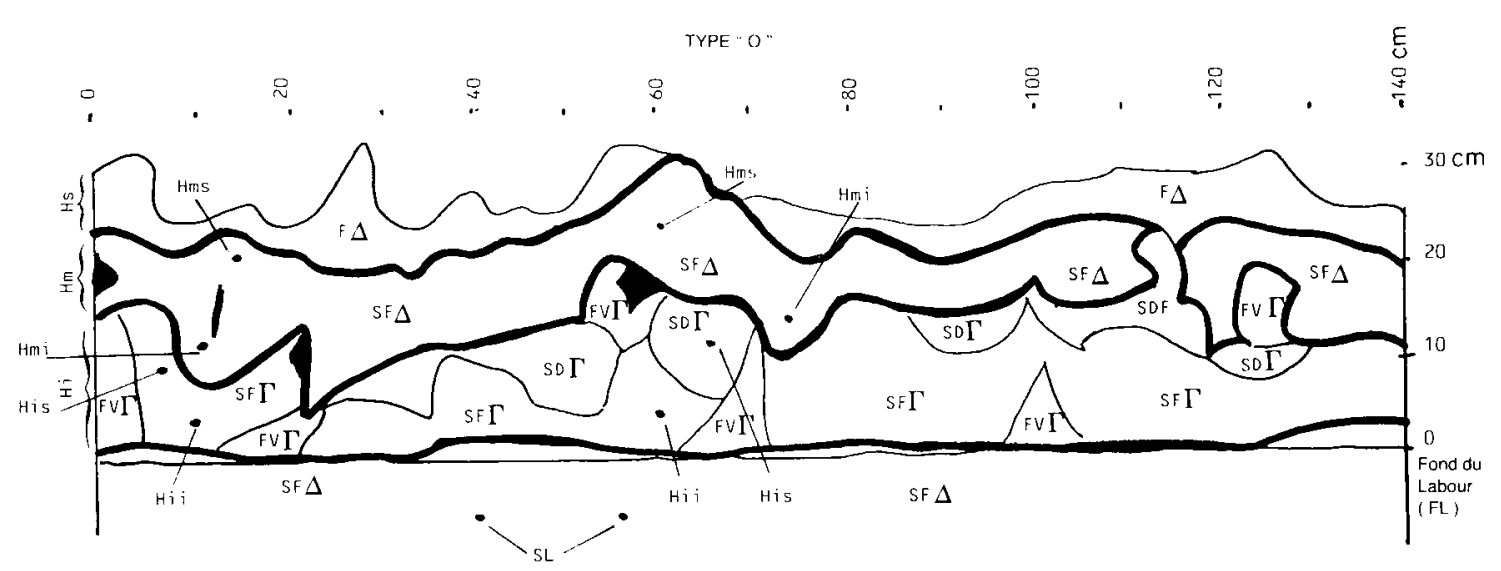

TYPE " B "

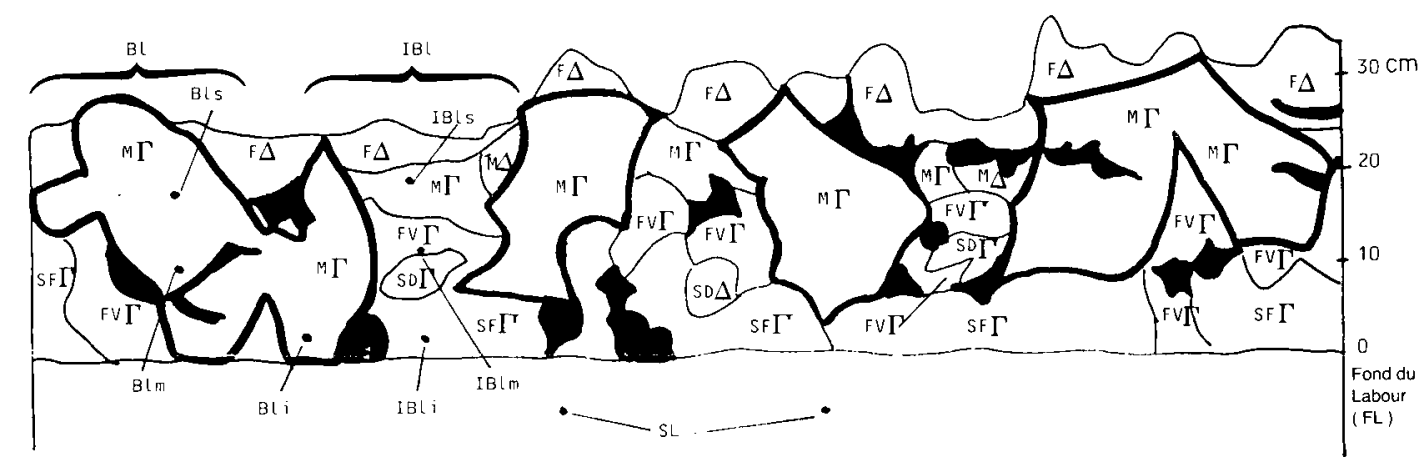

LEGENDE

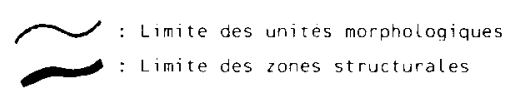

- Point de prélèvement d'humidité
: Vide centimétrique

Figure 1

Cartographie des deux types de couches labourées.

Mapping of two types of ploughed layer.

TABLEAU 2

Caractéristiques moyennes du sol étudié.

Mean characteristic features of the soil used.

\begin{tabular}{|c|c|c|c|c|c|c|c|}
\hline Profondeur & $\begin{array}{c}\mathrm{A} \\
<2 \mu\end{array}$ & $\begin{array}{c}1 \\
2-20 \mu\end{array}$ & $\underset{20-50 \mu}{\mathrm{L}}$ & $\stackrel{\mathrm{s}}{\mathrm{s} 0-100 \mu}$ & $\underset{200-2000 \mu}{S}$ & $\begin{array}{c}\text { Matière } \\
\text { organique } \%\end{array}$ & $\begin{array}{c}\text { Carbonates } \\
\%\end{array}$ \\
\hline Couche labourée & 27,4 & 26,4 & 32,3 & 10,2 & 4,1 & 3,1 & 3,0 \\
\hline $25-50 \mathrm{~cm}$ & 27,5 & 24,6 & 33,0 & 10,7 & 4,2 & 2,4 & - \\
\hline $50-85 \mathrm{~cm}$ & 26,1 & 25,3 & 33,9 & 10,5 & 4,2 & 1,8 & - \\
\hline
\end{tabular}

Note : Les horizons sous la couche labourée sont très filtrants et le siège d'une forte activité biologique (vers de terre).

Le sol, avant labour, ne portait pas de résidus végétaux ; par l'emploi de désherbants totaux, il a été maintenu sans végétation. Les 2 couches labourées ont subi des alternances d'humectation par les pluies et de dessiccation. Nous avons suivi l'évolution de l'état hydrique au cours de ces dernières, pendant la période allant du 10 mars à la fin juin.

$\mathrm{Au}$ cours de la période de mesures, l'évolution structurale sous l'action des agents climatiques a été faible. La caractérisation morphologique des états structuraux que nous allons présenter ci-dessous peut donc être considérée comme représentative des situations étudiées pendant toute la période des mesures.

\section{A. Caractérisation des états structuraux de la couche labourée}

Les états structuraux ont été caractérisés comme indiqué ci-dessus. On trouvera sur la figure 1 la représentation cartographique des 2 couches labourées. On peut y constater que les discontinuités majeures de la couche de type $\mathrm{O}$ sont subhorizontales et celles de la couche $\mathrm{B}$ subverticales. Aussi distingue-t-on, dans $\mathrm{O}$, des horizons ( $\mathrm{Hs}, \mathrm{Hm}$ et $\mathrm{Hi}$, cf. fig. 1) et, dans $\mathrm{B}$, des blocs (B1) et des interblocs (IB1) ; ces derniers sont constitués d'unités morphologiques très dissemblables les unes des autres. 


\section{B. Caractérisation des états hydriques de la couche travaillée}

Nous avons suivi l'évolution des états hydriques de la couche labourée sous l'effet des séquences climatiques selon un pas de temps variable allant d'un jour (juste après pluie) à une semaine, par des mesures de teneur pondérale et de tensiométrie.

\section{Mesures des teneurs pondérales en eau in situ}

L'humidité moyenne de la couche labourée a été obtenue en faisant la moyenne des teneurs en eau de 4 carottes (diamètres $7 \mathrm{~cm}$ ), distantes de $12 \mathrm{~cm}$, dans une direction perpendiculaire au sens du labour sur une largeur correspondant à 2 socs de charrue.

La mesure de l'humidité des différentes unités morphologiques a posé un problème. Faute de temps, il n'était pas possible, à chaque jour de prélèvement, de repérer chacune d'entre elles ; nous avons donc distingué les discontinuités structurales les plus marquées et fait des regroupements d'unités morphologiques en zones structurales comme indiqué sur la figure 1 . Le tableau 3 donne, pour $\mathrm{O}$ et $\mathrm{B}$, la répartition des unités morphologiques dans chacune d'elles. Les prélèvements d'échantillons ont été pratiqués dans ces zones à plusieurs niveaux dont les cotes, repérées par rapport au fond du labour, ont les valeurs indiquées sur la figure 1. A chaque date de prélèvement, la valeur retenue par cote, pour une zone structurale donnée, est la moyenne de 2 mesures.

La méthode utilisée étant destructrice, ces mesures sont faites chaque fois sur des placettes différentes, ce qui entraîne une erreur d'échantillonnage à l'échelle métrique. A 2 reprises, au cours de la campagne de mesure, nous avons déterminé simultanément les humidités sur 6 placettes réparties dans le dispositif, ce qui nous a permis de calculer les intervalles de confiance (tabl. 4).

\section{TABLEAU 4}

Intervalle de confiance, au risque $\alpha=0,05$, des mesures d'humidité dans les labours (en \% d'humidité pondérale).

Confidence limits (with risk $\alpha=0.05$ ) of water content values in ploughed layer.

\begin{tabular}{ccccc}
\hline \hline $\begin{array}{c}\text { Type de } \\
\text { labour }\end{array}$ & $\begin{array}{c}\text { Humidité } \\
\text { moyenne de } \\
\text { la couche }\end{array}$ & $\begin{array}{c}\text { Surface } \\
\mathrm{Hs}\end{array}$ & $\mathrm{Hm}$ & $\mathrm{Hi}$ \\
\hline $\mathrm{O}$ & $\begin{array}{l} \pm 0,5 \\
\pm 0,7\end{array}$ & $\pm 0,8$ & $\pm 0,9$ & $\pm 0,9$ \\
$\mathrm{~B}$ & \pm 0 & - & - \\
\hline
\end{tabular}

\section{Détermination tensiométrique de la succion (h) in situ}

La mise en place de tensiomètres dans les unités morphologiques d'une couche labourée est délicate. Elle implique qu'on utilise une petite bougie fixée à un cylindre rigide. La nécessité de repérer les unités morphologiques dans lesquelles on veut suivre le potentiel hydrique oblige à introduire des bougies horizontalement, à une dizaine de $\mathrm{cm}$ de la paroi d'une tranchée d'observation creusée pour l'installation de l'appareil, puis rebouchée soigneusement. On vérifie au moment de retirer le dispositif qu'il y a continuité spatiale de l'état structural entre la paroi de la tranchée, réouverte pour l'occasion, et le lieu d'implantation de la bougie. Dans le labour $O$, sur un site, les mesures tensiométriques ont été réalisées à 6 , 11,22 et $32 \mathrm{~cm}$, ce qui permet de suivre l'évolution du profil de charge hydraulique $\mathrm{H}$. A chaque cote $(\mathrm{z})$, $\mathrm{H} \mathrm{cm}=-\mathrm{zcm}+\mathrm{h} \mathrm{cm}$ (cf. tabl. 5).

TABLEAU 3

Répartition des unités morphologiques dans les différents types de labour (en \%). Distribution of morphological units in different ploughed layers (\%).

\begin{tabular}{|c|c|c|c|c|c|}
\hline \multicolumn{3}{|c|}{ Type «O » } & \multicolumn{3}{|c|}{ Type $\langle B »$} \\
\hline \multicolumn{3}{|c|}{ Orientation générale : Sub-horizontale } & \multicolumn{3}{|c|}{ Orientation générale : Sub-verticale } \\
\hline Zone & $\begin{array}{c}\text { Unité } \\
\text { morphologique }\end{array}$ & $\%$ & Zone & $\begin{array}{c}\text { Unité } \\
\text { morphologique }\end{array}$ & $\%$ \\
\hline Horizon de surface (Hs) & $\mathrm{F} \Delta$ & 21,6 & Surface & $\mathrm{F} \Delta$ & 9,2 \\
\hline Horizon moyen $(\mathrm{Hm})$ & $\mathrm{SF} \Delta$ & 23,4 & Blocs sub-verticaux & $\mathrm{M} \Gamma$ & 42,0 \\
\hline \multirow[t]{3}{*}{ Horizon inférieur (Hi) } & $\mathrm{SD} \Gamma$ & 9,1 & Inter-blocs & $\mathrm{SD} \Delta$ & 6,4 \\
\hline & SFT & 28,0 & & $\mathrm{SD} \Gamma$ & 5,4 \\
\hline & FVГ $\left(^{1}\right)$ & 9,3 & & SFT & 11,4 \\
\hline Mottes écrasées en fond de & $\mathrm{Mr}$ & 70 & & $\mathrm{~F} \Gamma$ & 4,6 \\
\hline labour & MII & 1,0 & & $\mathrm{FV} \Gamma\left(^{1}\right)$ & 12,1 \\
\hline Vides centimétriques & $V\left({ }^{l}\right)$ & 1,5 & Vides centimétriques & $V(1)$ & 8,9 \\
\hline
\end{tabular}

Sous la couche labourée : SF $\Delta$

( $\left.{ }^{1}\right)$ Les vides sont quantifiés en tant que tels lorsqu'ils occupent une surface de plusieurs $\mathrm{cm}^{2}$ sur le profil. FV signifie « Structure fragmentaire avec vides importants "). 


\section{Détermination au laboratoire des fonctions : humidité pondérale $=\mathbf{f}(\mathrm{pF})$ et des systèmes poreux}

Nous avons déterminé au laboratoire la loi de variation de l'humidité en fonction d'une contrainte de type succion hydrostatique sur des échantillons non remaniés d'unités morphologiques de 2 à $4 \mathrm{~cm}^{3}$. L'étude n'a porté que sur celles des unités que nous avons pu prélever dans le profil sans perturbation, à savoir $\mathrm{M} \Delta, \mathrm{SD} \Delta, \mathrm{SF} \Delta, \mathrm{M} \Gamma$ et $\mathrm{SD} \Gamma$.

Au-dessous de succions de 40 bars, nous avons utilisé le dispositif d'ultrafiltration mis au point par TESSIER \& BERRIER (1979) et, au-dessus de 40 bars, une gamme de solutions salines permettant d'obtenir, en enceintes fermées, des atmosphères à humidité relative très faibles. La relation $w \%=f(p F)$ a été établie en régime de dessiccation (le $\mathrm{pF} 0,3$ a été cependant obtenu par humectation car, à humidité très élevée, les échantillons ne sont pas transportables sans déformation).

Le volume des systèmes poreux que constituent les différentes unités morphologiques a été mesuré sur des échantillons humides par la méthode au pétrole (MONNIER et al., 1973).
III. EFFET DE L'ÉTAT STRUCTURAL DE NIVEAU 2 SUR LE COMPORTEMENT HYDRIQUE D'UNE COUCHE LABOURÉE

\section{A. Les humidités pondérales in situ}

L'étude de l'effet de l'état structural de niveau 2 sur le comportement hydrique de la couche labourée a été réalisé sur $\mathrm{O}$, car les interblocs de la couche $\mathrm{B}$ étaient trop hétérogènes pour qu'on puisse y faire facilement des prélèvements d'humidité correspondant à un seul type morphologique. Sur la figure 2 sont reportées les valeurs d'humidité pondérales des différentes zones structurales de la couche de type $O$.

Hormis les $5 \mathrm{~cm}$ de surface, dont l'humidité est fluctuante au cours du temps, on observe, sur la période de mesures, une bonne stabilité des teneurs en eau des prélèvements réalisés aux cotes inférieures. En comparant l'évolution hydrique des points de cote voisine, on constate :

1) Qu'au sein de chacun des 2 horizons, $\mathrm{Hm}$ (constitué de SD $\Delta$ ) et Hi (essentiellement constitué de SFT), la différence de teneur en eau est très faible entre points de cote distants de 7 à $9 \mathrm{~cm}$.
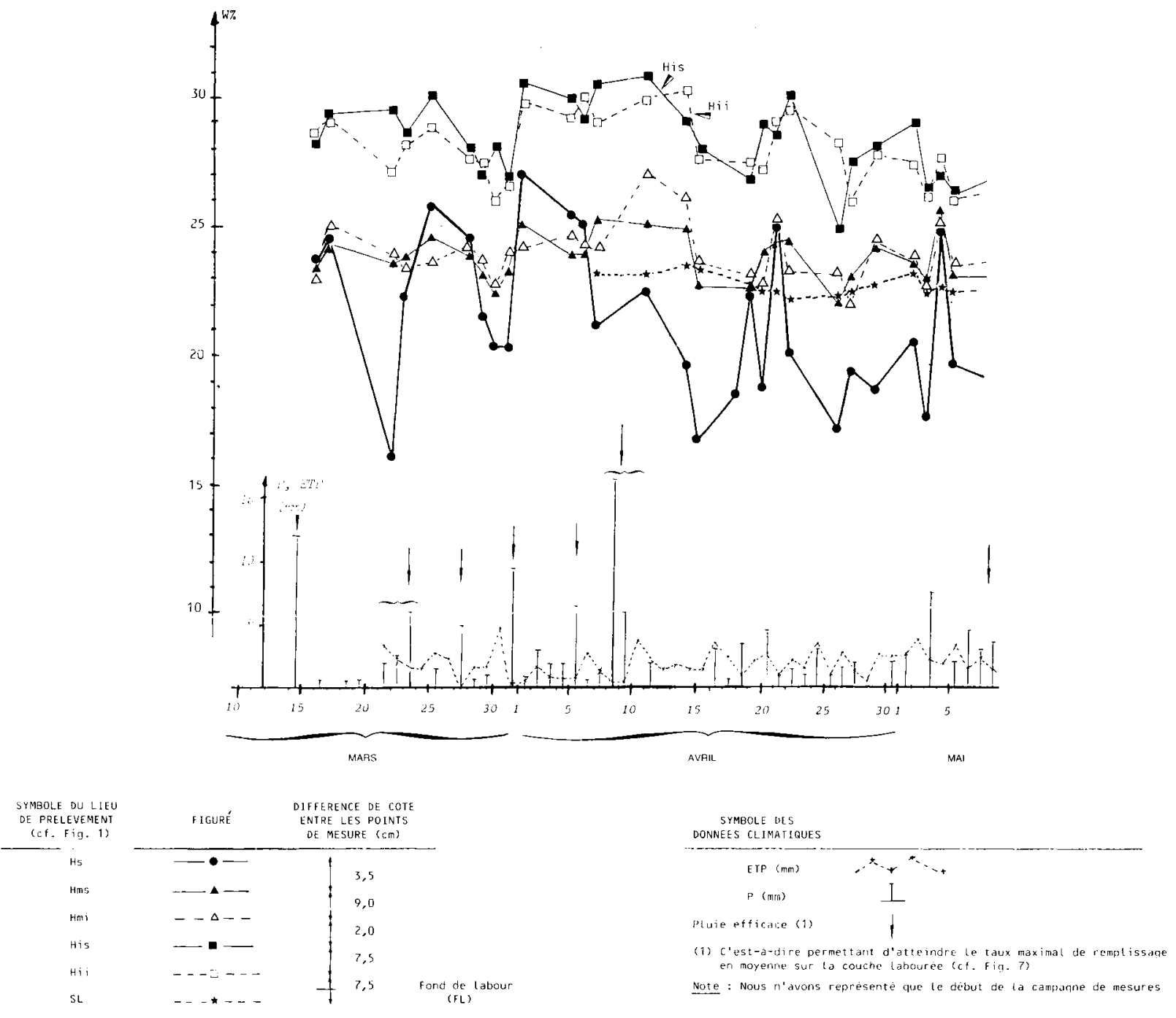

Figure 2

Evolution de l'humidité pondérale (w\%) dans les différentes zones structurales du type «O».

Ponderal water content ( $w \%$ ) in structural zones of type « $O$ ». 
2) Que, de part et d'autre des interfaces $\mathrm{Hm}$-Hi et $\mathrm{Hi}$-SL, elle est au contraire élevée ; les points $\mathrm{Hmi}$ et His, distants en moyenne de $2 \mathrm{~cm}$, et les points Hii et $\mathrm{SL}$, distants de $7 \mathrm{~cm}$, présentent une différence de teneur portant sur plusieurs points d'humidité (3 à 4 p. 100 environ).

On peut donc parler d'une double discontinuité hydrique de part et d'autre de Hi. Cet horizon (SF principalement) reste constamment plus humide que les 2 couches qui l'entourent, constituées de SF $\Delta$.

Que le fond du labour soit relativement plus humide que les autres horizons est un fait d'observation courante au printemps, souvent attribuable à une différence de conductivité hydraulique entre la couche labourée et celle sous-jacente, mais, dans la situation étudiée, cette explication ne paraît pas suffisante car :

1) La couche SL possède une grande perméabilité.

2) S'il y avait un effet de freinage de la circulation de l'eau au fond du labour, on observerait, après les épisodes pluvieux importants, un gradient hydrique dans l'horizon Hi. Or la différence d'humidité entre His et Hii (distants en moyenne de $7,5 \mathrm{~cm}$ ) reste toujours inférieure à l'intervalle de confiance estimé $(2 \times 0,9$ p. 100), tandis que celle observée entre $\mathrm{Hmi}$ et His (distants de $2 \mathrm{~cm}$ ) lui est supérieure.

Il existe donc bien des discontinuités de teneur pondérale en eau de part et d'autre des faces de discontinuités structurales.

\section{B. Les profils de charge à partir des mesures tensio- métriques in situ}

Les mesures tensiométriques réalisées in situ permettent d'établir des profils de charge hydraulique pour chaque date de mesure. On constate, tout au moins pendant toute la période de fin avril-début mai où l'ETP est encore relativement faible - voisine de $2 \mathrm{~mm} / \mathrm{j}$ - qu'entre les zones structurales $\mathrm{Hm}$, Hi et $\mathrm{Sl}$, il y a continuité de charge puisque, du haut en bas du profil, les différences de charge $\mathrm{H}$ sont du même ordre de grandeur pour une même variation de cote $\mathrm{z}$ (fig. 3).

L'ensemble de ces observations sur les profils hydriques et les profils de charge montre qu'à des énergies de succion quasi identiques, (points de cotes très voisines) peuvent correspondre des humidités pondérales très différentes si ces 2 points se situent dans des unités morphologiques distinctes. Ceci suggère l'existence d'un effet de l'état structural au niveau 2 sur la rétention de l'eau. Dans la mesure où nous pourrons retirer des unités morphologiques du profil cultural et les transporter sans les déformer, nous allons vérifier et analyser cet effet au laboratoire.

\section{Les relations pF-Humidité établies en laboratoire sur des unités morphologiques}

Les mesures de volume à l'état humide permettent de calculer la porosité (exprimée en indice des vides eT) (tabl. 5), connaissant la masse volumique solide $\rho s$ (la valeur mesurée de $\rho s$ est de $2,68 \mathrm{~g} / \mathrm{cm}^{3}$ ). Le tableau 6 donne l'indice des vides total, le mode d'assemblage et l'état interne des différents systèmes poreux que constituent les unités morphologiques étudiées. Il montre clairement que, pour un même mode d'assemblage, il y a une différence d'une classe de porosité selon la nature de l'état interne.

Les relations $\mathrm{pF}$ - Humidité de chaque unité sont données sur la figure 4 . Elles confirment les résultats de travaux antérieurs, à savoir l'effet de l'état structural sur ces relations. CHANG (1968), STAKMAN et al.,

E
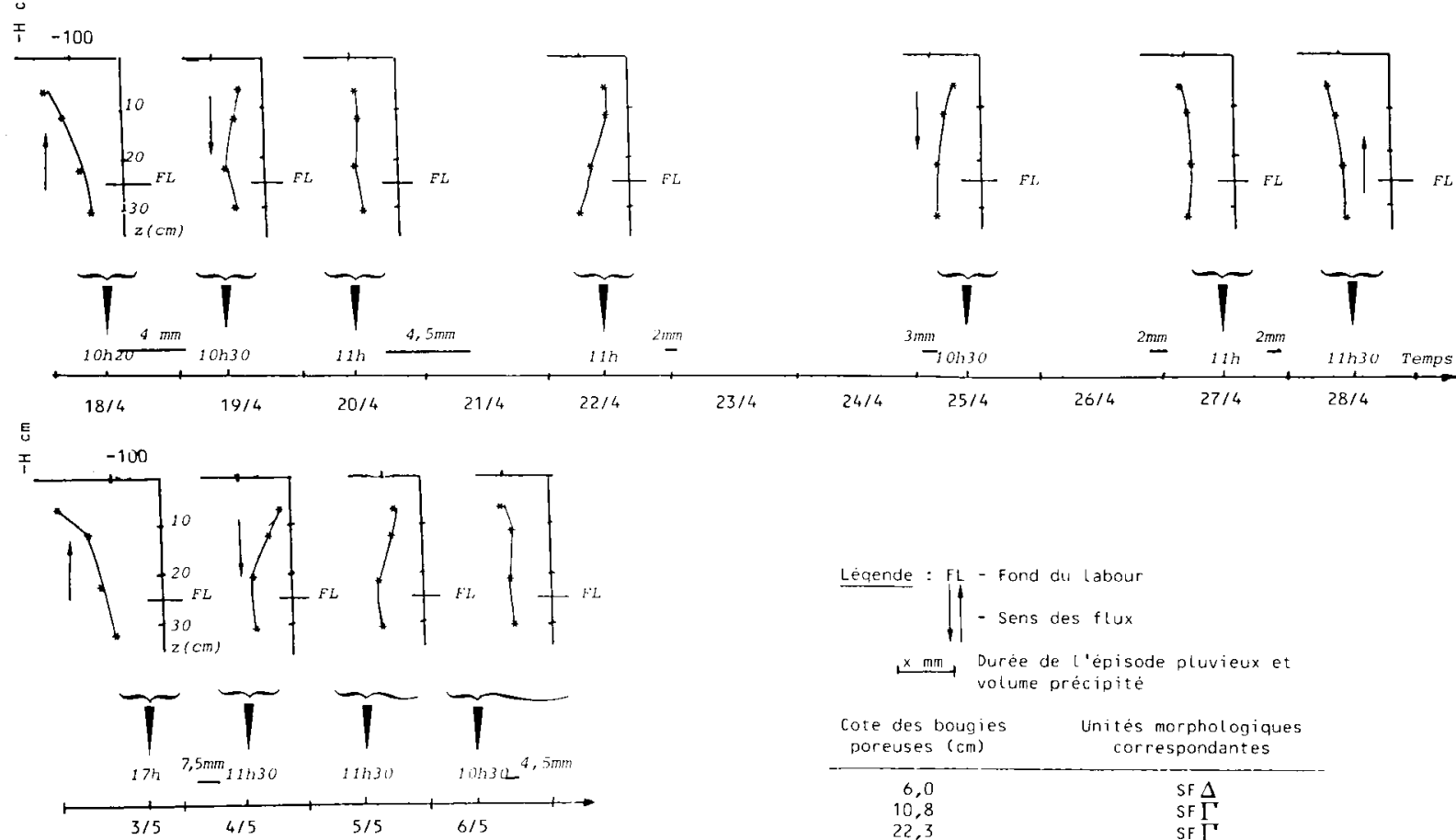

Figure 3

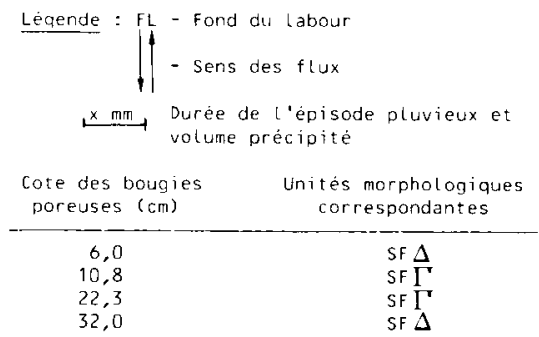

Evolution des profils de charge $(H \mathrm{~cm})$ dans la couche labourée de type « $O$ ».

Hydraulic load profile $(H \mathrm{~cm})$ in ploughed layer $O$. 
TABLEAU 5

Symboles utilisés pour caractériser l'état hydrique et l'espace poral. Symbols used to characterize hydric state and porosity.

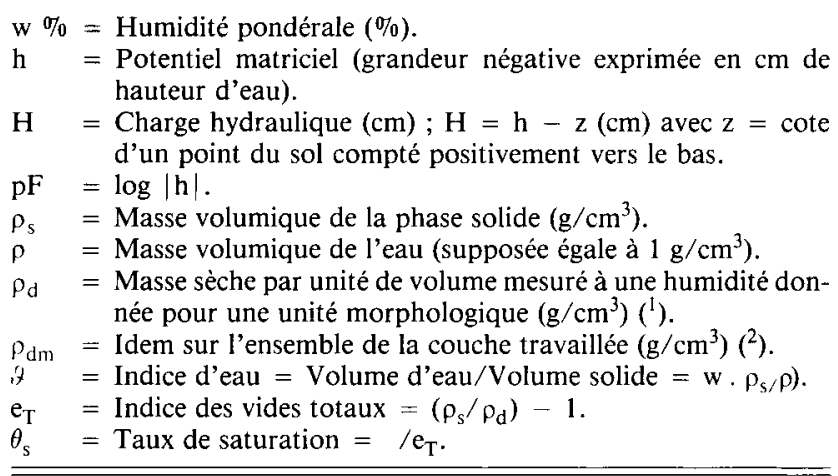

(1) Mesurée par la méthode de l'anneau ou par porosité de mottes. (2) Mesurée par la méthode du cylindre, les cotes étant repérées par aspérimétrie (PAPY, 1984).

\section{TABLEAU 6}

Porosité totale (exprimée en indice des vides totaux $e_{T}$ ) des différentes unités morphologiques (teneur en eau $=0,20 \mathrm{~g} / \mathrm{g}$ ).

Total porosity (in total void ratio $e_{T}$ ) of different morphological units (water content : $20 \mathrm{~g} / \mathrm{g}$ ).

\begin{tabular}{|c|c|c|c|}
\hline Classe de porosité & 0 & 1 & 2 \\
\hline $\mathrm{e}_{\mathrm{T}}$ & $<0,3$ & $0,3-1,9$ & $1,9-3,0$ \\
\hline $\begin{array}{l}\text { Type d'unité morpho- } \\
\text { logique }\end{array}$ & $\mathrm{M} \Delta$ & $\begin{array}{l}\mathrm{M} \Gamma \\
\mathrm{SD} \Delta\end{array}$ & $\begin{array}{l}\mathrm{SD} \Gamma \\
\mathrm{SF} \Delta\end{array}$ \\
\hline
\end{tabular}

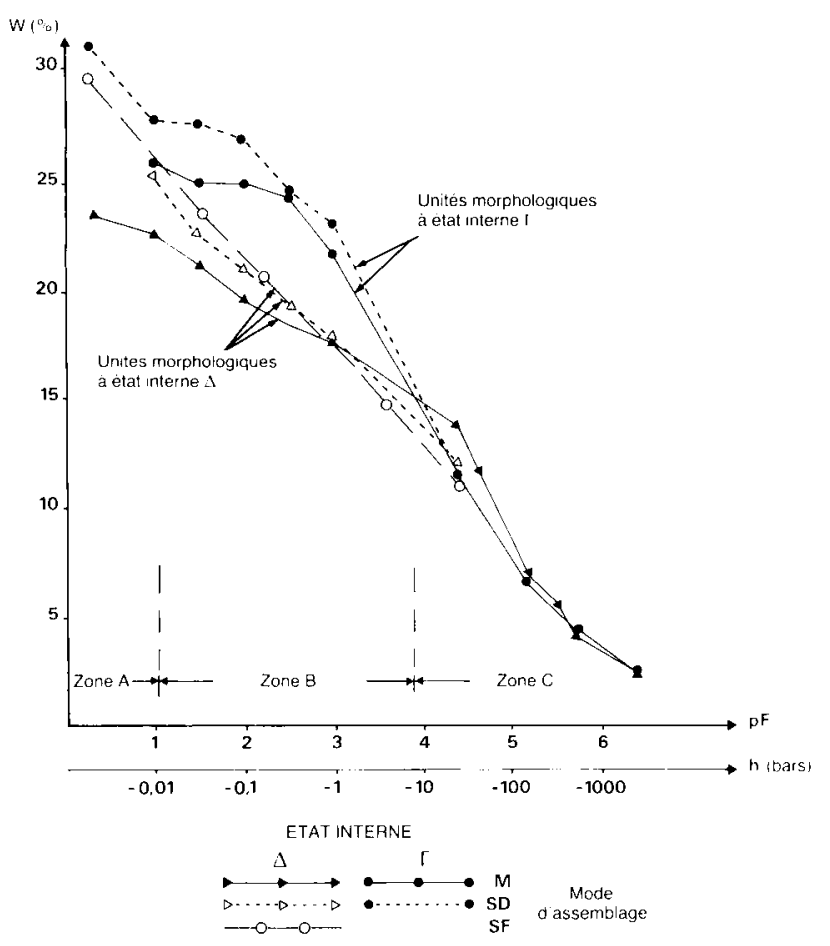

Figure 4

Relation pF-Humidités pondérales (w\%) pour quelques unités morphologiques.

pF-Water content (w\%) relationship for some morphological units.
(1969), ont montré qu'aux très faibles succions les mottes ayant une forte porosité interagrégats contenaient plus d'eau que les mottes plus massives. CRONEY \& COLEMAN (1954), CHANG (1968), WARKENTIN (1971), GRIMALDI (1981), ont établi que les agrégats les plus compacts contiennent moins d'eau aux faibles succions et davantage aux plus fortes.

Mais le fait de caractériser l'état structural des unités morphologiques à la fois par l'état interne des éléments structuraux et par le mode d'assemblage permet d'analyser plus finement ces relations (fig. 4). On peut distinguer 3 zones:

- Zone A : pour pF $<1$; c'est la porosité totale (celle de l'état interne et celle de l'assemblage) qui classe les courbes et, dans le cas limite où les échantillons sont saturés d'eau, le classement des porosités totales est bien celui des teneurs en eau.

- Zone $\mathrm{B}: 1<\mathrm{pF}<4$; dans cette gamme de $\mathrm{pF}$, la nature de l'état interne des éléments structuraux $(\Delta$ ou $\Gamma$ ) discrimine les courbes en 2 familles distinctes : à même énergie de fixation, ce sont les unités morphologiques à état interne $\Gamma$ qui contiennent le plus d'eau.

Dans cette gamme de $\mathrm{pF}$, nous avons comparé les indices de vides totaux $\left(\mathrm{e}_{\mathrm{T}}\right)$ et les indices d'eau $(\vartheta)$ (cf. tabl. 5 pour la définition des indices). Pour chacune des unités morphologiques, ces indices sont reportés sur la figure 5. Les graphiques représentatifs des états internes ( $\mathrm{M} \Delta$ et $\mathrm{M} \Gamma$ ) mettent bien en évidence que, contrairement aux éléments $\Gamma$, les éléments $\Delta$ sont presque totalement saturés jusqu'à $p F 3$, où le taux de saturation $\vartheta / \mathrm{e}_{\mathrm{T}}$ est encore égal à 0,92 .

Sur les graphiques correspondant aux structures $\mathrm{SD} \Delta, \mathrm{SF} \Delta$ et $\mathrm{SD} \Gamma$, nous avons reporté respectivement les courbes d'indices des vides totaux des unités $\mathrm{M} \Delta$ et $\mathrm{M \Gamma}$. Ainsi peut-on distinguer, pour chaque valeur de $\mathrm{pF}$, la porosité intra-élément structural de la porosité extra due au mode d'assemblage et, par suite, analyser comment l'eau se répartit dans les deux.

Dans la gamme de $\mathrm{pF}$ de 1 à 4 , on constate que les indices d'eau des unités $\mathrm{SD} \Delta, \mathrm{SF} \Delta$ et $\mathrm{SD} \Gamma$, avoisinent les valeurs d'indice de vides totaux de $\mathrm{M} \Delta$ et $\mathrm{M} \Gamma$ respectivement, ce qui est la traduction de l'effet prépondérant de l'état interne sur la rétension en eau. A noter que l'allure des relations $\vartheta=\mathrm{f}(\mathrm{pF})$ varie d'une famille à l'autre : l'indice d'eau est invariant entre pF 1 et 3 pour la famille à éléments $\Gamma$ tandis que sa variation est continue dans celle à éléments $\Delta$. Ceci peut s'interpréter par une différence de répartition de classes de taille de pores (KLUTE, 1982) qui a été effectivement mise en évidence sur ce même sol par porosimétrie au mercure (FIÈs, travaux en cours).

- Zone $\mathrm{C}: \mathrm{pF}>4$ (fig. 4). Au fur et à mesure que le $\mathrm{pF}$ augmente, on constate une légère tendance à l'inversion de l'effet " état interne » $(\Delta$ contient un peu plus d'eau que $\Gamma$ ) puis une atténuation progressive de cet effet conformément aux résultats d'autres auteurs cités plus haut.

\section{Discussion}

Ainsi, selon le niveau énergétique, ce sont les modes d'assemblage entre éléments structuraux ou la nature 

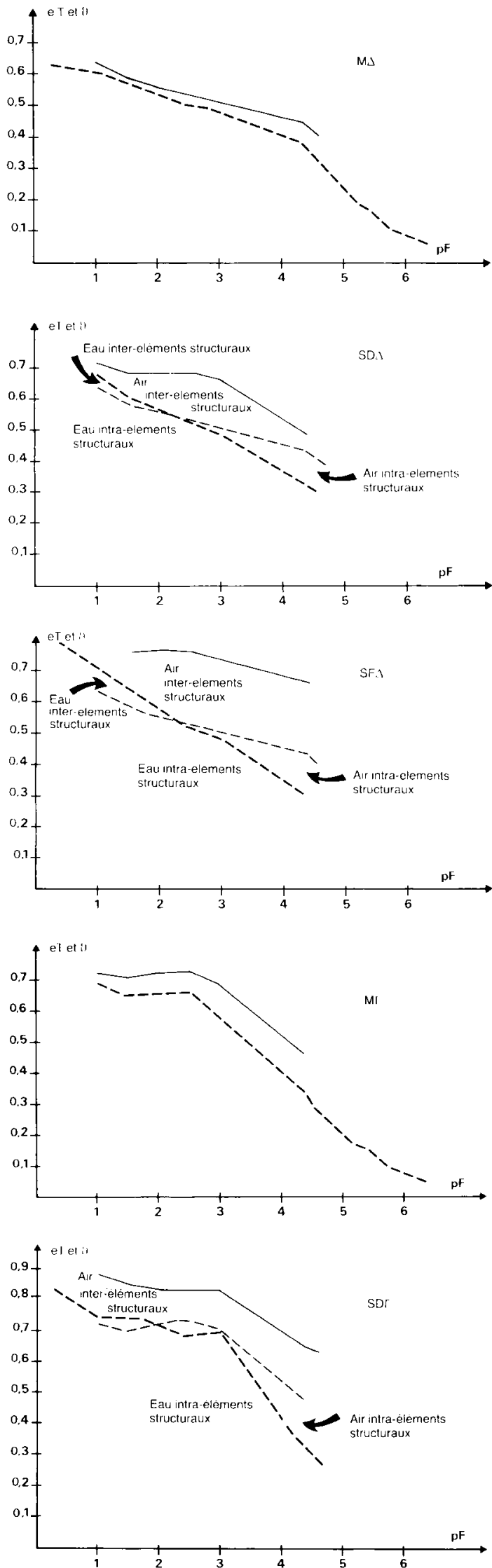

Figure 5

Variation de l'indice de vides totaux (eT-) et de l'indice d'eau (1...) de quelques unités morphologiques en fonction $d u p F$.

Variation of total void ratio ( $\mathrm{eT} \longrightarrow$ ) and water ratio ( $\left(\varphi_{----)}\right)$of some morphological units with $\mathrm{pF}$.

Note : On graphs corresponding to SDA, SFA et $S D \Gamma$, curves of total void ratio of $M \Delta$ and $M \Gamma$ are respectively reported $(-\cdots)$. même de l'état interne de ces éléments $(\Delta$ ou $\Gamma$ ) qui déterminent les rétentions de l'eau.

Les mesures tensiométriques in situ ont montré que, pendant la période d'observation du début printemps, au-dessous des quelques $\mathrm{cm}$ de surface, le $\mathrm{pF}$ reste entre 1 et 3 , ce qui correspond, à l'équilibre, aux humidités respectives suivantes : 26 et 18 p. 100 pour $\mathrm{SF} \Delta, 28$ et 23 p. 100 pour $\mathrm{SD} \Gamma$. On retrouve bien l'ordre de grandeur des humidités que nous avons signalé respectivement pour $\mathrm{Hm}$ (constitué de $\mathrm{SF} \Delta$ ) et $\mathrm{Hi}$ (constitué principalement de SD $\Gamma$ ). Les discontinuités de teneur pondérale en eau, observées dans le profil de la couche $O$, sont bien liées à des capacités de rétention de l'eau différentes des unités morphologiques.

\section{EFFET DE L'ÉTAT STRUCTURAL DE NIVEAU 3 SUR LE COMPORTEMENT HYDRIQUE DE LA COUCHE LABOURÉE}

L'effet structural de niveau 3 est analysé par comparaison de l'évolution des teneurs moyennes en eau des 2 types de couche labourée, $O$ et $B$. La figure 6 montre que, tout au long de la campagne de mesures, la couche $O$ est plus humide que B. Mais cet écart existe depuis le début des observations. Sa persistance au cours du printemps ne résulte-t-elle pas de la différence initiale? Pour répondre à cette question nous avons donc examiné si les pluies ont été suffisantes pour assurer le remplissage maximum de la couche.

\section{A. Mise en évidence d'une différence de taux maxi- mum de remplissage par les pluies}

Pour mettre en évidence un éventuel taux maximum de remplissage, on détermine l'humidité après les différents épisodes pluvieux classés d'après leur capacité à remplir la couche labourée. Pour ce faire, on calcule pour chaque pluie la teneur en eau r p. 100 telle que :

$$
\mathrm{r}=\mathrm{x}+\mathrm{y} \text {, avec }
$$

$\mathrm{x}(\mathrm{p} .100)=$ teneur moyenne en eau juste avant pluie,

$y(p .100)=$ surcroît de teneur, sous hypothèse que la couche labourée retienne toute l'eau reçue, c'est-àdire qu'en tombant sur une surface unitaire de $1 \mathrm{~mm}^{2}$, une pluie de $\mathrm{P} \mathrm{mm}$ reste dans le volume de $\mathrm{p} \mathrm{mm} \times 1$ ( $p$ étant la profondeur de la couche). La masse de sol qui correspond à ce volume est $: p \times \rho_{\mathrm{dm}}$. Il vient alors :

$$
y\left(\text { p. 100) }=\frac{P}{p \cdot \rho_{d m}} \times 100 .\right.
$$

Sur l'ensemble de la campagne d'observations, nous disposons de 7 mesures réalisées dans un délai de 6 à $12 \mathrm{~h}$ après l'arrêt des précipitations et pendant lequel ETP peut être considérée comme négligeable. Nous avons reporté ces valeurs en fonction de la valeur $r$ de chaque épisode pluvieux en distinguant ces derniers 


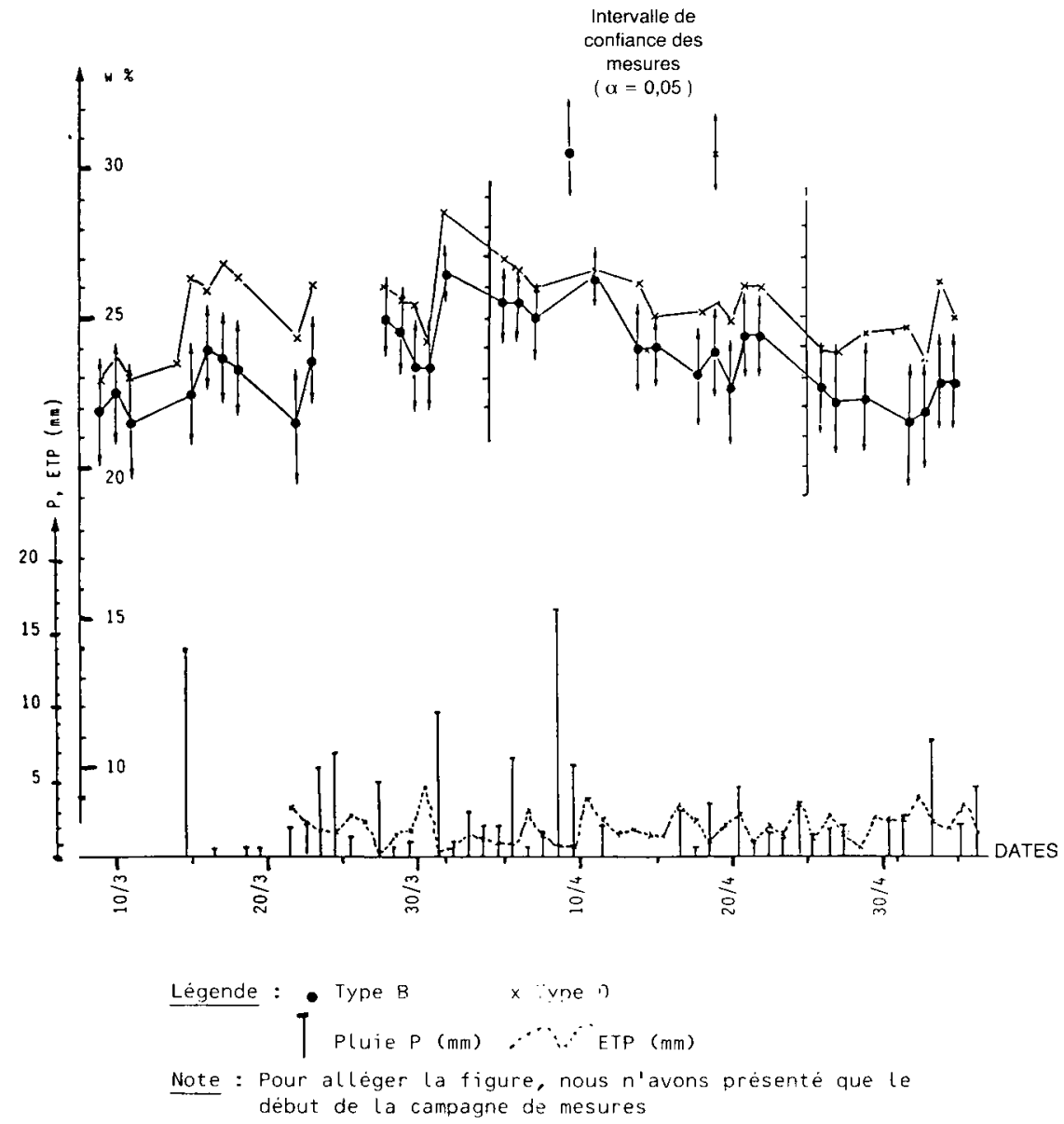

Figure 6

Evolution de l'humidité moyenne pondérale (w\%) des deux types de couches labourées.

Mean ponderal water content of the two types of ploughed layers.
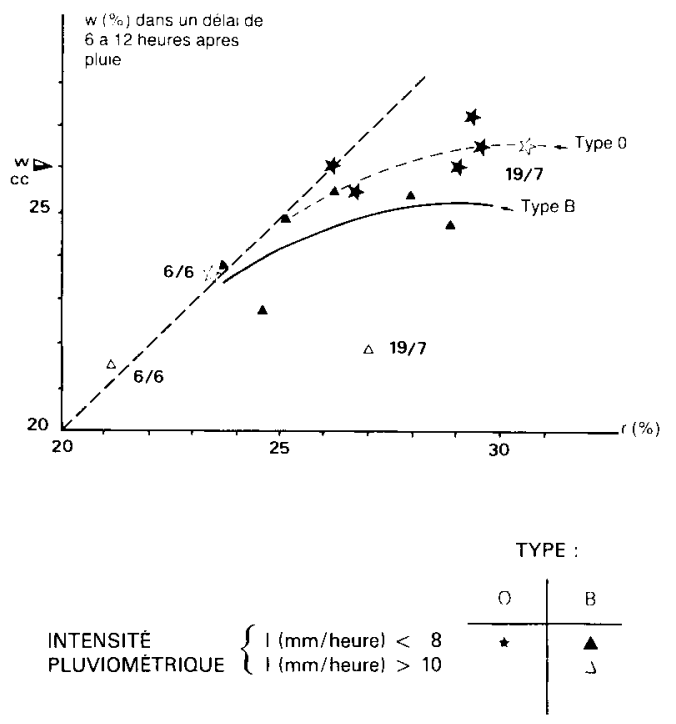

W cc : Humidité à la capacité au champ calculée

Figure 7

Détermination de l'humidité pondérale maximale retenue quelques heures après pluie par les deux types « $O$ » et « $B$ ».

Establishment of maximum water content in two ploughed layers $O$ and $D$, a few hours after rain. selon l'intensité pluviométrique (fig. 7). Nous constatons :

1) Qu'il existe bien une valeur maximale de remplissage pour chacune des 2 couches labourées: elle est plus élevée d'un point d'humidité pour $\mathrm{O}$ que pour $\mathrm{B}$, écart supérieur à l'intervalle de confiance (tabl. 4). Remarquons que, dans le cas de la couche de type O, elle correspond à la valeur calculée de l'humidité à la capacité au champ en fonction des résultats de l'analyse granulométrique: W cc (HÉNIN et al., 1969).

2) Qu'à la différence du type $O$, le type B n'a pas le même comportement selon l'intensité pluviométrique ; nous avons mis ce phénomène en évidence en étudiant la réaction des 2 couches labourées à une pluie orageuse de $28 \mathrm{~mm}$ en $2 \mathrm{~h}$, survenue très tardivement le 19 juillet : elle a ramené $\mathrm{O}$ à son maximum de remplissage, mais pas B.

Si l'on porte l'humidité moyenne de la couche labourée en fonction du temps écoulé après les pluies susceptibles de remplir la couche aux valeurs maximales de remplissage (que nous appellerons pluies efficaces), on obtient la figure 8. D'un type de couche labourée à l'autre, les variances et les pentes ne sont pas significativement différentes. Les ordonnées à 


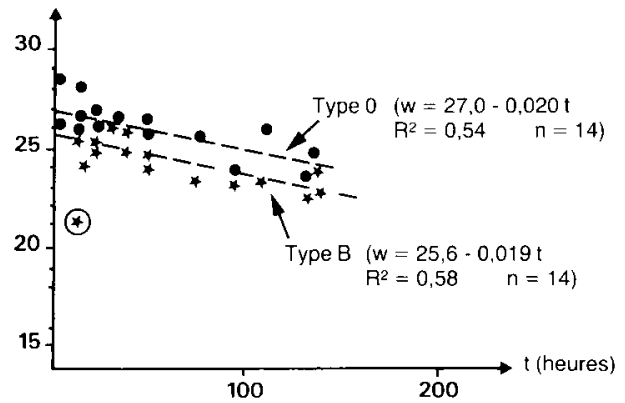

$\star$ Point correspondant à l'orage du 19/07. II n'a pas été pris en compte dans l'équation de régression.

Figure 8

Evolution de l'humidité moyenne de la couche labourée en fonction du temps écoulé après une pluie efficace. (Type «O»:- Type " $B$ »: $\star$ ).

Mean water content of ploughed layer in relation to time after efficient rain.

l'origine le sont, par contre, au seuil $\alpha=0,005$. Aussi l'écart de teneur en eau enregistré tout au long de la campagne (fig. 6) est bien le résultat d'une différence de capacité à emmagasiner l'eau des pluies. C'est un effet de l'état structural de niveau 3 que nous allons maintenant analyser.

\section{B. Discussion de l'effet de l'état structural de niveau 3 sur l'humectation par les pluies}

Dans ses travaux sur le phénomène d'infiltration, FÉODOROFF (1965) montre que dans les systèmes poreux constitués d'ensembles compacts séparés de lacunes, l'eau, y circulant vite, imbibe peu la masse de terre. Par contre, un système poreux constitué de terre fine absorbe mieux l'eau en cours d'infiltration. Pour que cette hypothèse puisse être retenue dans l'explication de la différence que nous venons de mettre en évidence entre couches $\mathrm{O}$ et $\mathrm{B}$, elle doit se traduire par les 2 conséquences suivantes:

1) Peu de temps après une pluie efficace, pour 2 points de même cote, l'état hydrique doit être plus sec en zone compacte qu'en zone meuble.

2) La vitesse de circulation de l'eau (qui est fonction de l'intensité pluviométrique) doit accentuer les différences précédentes.

Puisque nous venons de montrer que, dans la gamme des états concernés, une zone compacte avait à l'équilibre une teneur pondérale en eau inférieure à celle d'une zone meuble, c'est une différence d'état énergétique de l'eau qu'il faut mettre en évidence entre zones compactes et meubles et non simplement de teneur en eau, pour valider l'hypothèse. N'ayant pas de tensiomètre in situ dans la couche $\mathrm{B}$, nous allons utiliser les relations $\mathrm{pF}-$ Humidité de la figure 4 sachant que, dans $\mathrm{O}, \mathrm{Hm}$ et $\mathrm{Hi}$ sont respectivement constitués de SF $\Delta$ et SDT et que, dans B, les blocs (B1) sont constitués de $\mathrm{M \Gamma}$. Les résultats du tableau 7 montrent bien :

1) La différence de $\mathrm{pF}$ attendue entre mesures réalisées à cote sensiblement identique $\mathrm{Hm}$ de $\mathrm{O}$ et $\mathrm{Blm}$ de $\mathrm{B}$, d'une part, $\mathrm{Hi}$ de $\mathrm{O}$ et Bli de $\mathrm{B}$, d'autre part.

2) La différence de comportement des labours selon l'intensité pluviométrique : la pluie du 19 juillet permet, dans $\mathrm{O}$, une imbibition des zones $\mathrm{Hm}$ et $\mathrm{Hi}$ aussi forte que les pluies efficaces de faible intensité, de même dans les interblocs (IB1) de B ; mais les blocs (B1) de B sont peu humectés.

L'ensemble de ces résultats peut bien s'interpréter par l'hypothèse d'une circulation préférentielle de l'eau en cours d'infiltration dans les lacunes et les interblocs de B.

TABLEAU 7

Etat hydrique des zones structurales des types « $O$ » et «B» quelques heures après des pluies efficaces. Effet de l'intensité pluviométrique. Water content of structural zones in ploughed layer $O$ and $B$ few hours after efficient rains. Effect ot the intensity of rain.

\begin{tabular}{|c|c|c|c|c|c|c|c|}
\hline & Intensité & $\begin{array}{c}\text { Zone } \\
\text { structurale }\end{array}$ & $\begin{array}{c}\text { Nombre } \\
\text { de } \\
\text { mesures }\end{array}$ & $\mathrm{w} \%$ & $\mathrm{~s}$ & $w-s ; w+s$ & $\mathrm{pF}\left({ }^{1}\right)$ \\
\hline \multirow{2}{*}{ 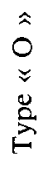 } & $\mathrm{I} \leqslant 4 \mathrm{~mm} / \mathrm{h}$ & $\begin{array}{l}\mathrm{Hm} \\
\mathrm{Hi}\end{array}$ & $\begin{array}{l}4 \\
4 \\
\end{array}$ & $\begin{array}{l}24,1 \\
29,0\end{array}$ & $\begin{array}{l}0,10 \\
0,8\end{array}$ & $\begin{array}{l}24,0 ; 24,2 \\
28,2 ; 29,8\end{array}$ & $\begin{array}{l}1,3 \\
1,0\end{array}$ \\
\hline & $\begin{array}{l}\mathrm{I}=14 \mathrm{~mm} / \mathrm{h} \\
\text { (orage du } 19 / 7 \text { ) }\end{array}$ & $\begin{array}{l}\mathrm{Hm} \\
\mathrm{Hi}\end{array}$ & $\begin{array}{l}2 \\
2\end{array}$ & $\begin{array}{l}24,5 \\
29,3\end{array}$ & & & $\begin{array}{l}1,2 \\
1,0\end{array}$ \\
\hline \multirow{2}{*}{ 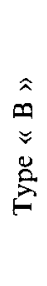 } & $\mathrm{I} \leqslant 4 \mathrm{~mm} / \mathrm{h}$ & $\begin{array}{l}\text { Blm } \\
\text { Bli } \\
\text { IBlm } \\
\text { IBli }\end{array}$ & $\begin{array}{l}4 \\
4 \\
4 \\
4\end{array}$ & $\begin{array}{l}25,1 \\
26,2 \\
26,6 \\
25,6\end{array}$ & $\begin{array}{l}1 \\
0,9 \\
0,8 \\
0,6\end{array}$ & $\begin{array}{l}24,1 ; 26,1 \\
24,3 ; 26,1\end{array}$ & $\begin{array}{l}2,6-1,0 \\
2,6-1,0\end{array}$ \\
\hline & $\begin{array}{l}I=14 \mathrm{~mm} / \mathrm{h} \\
\text { (orage du } 19 / 7 \text { ) }\end{array}$ & $\begin{array}{l}\text { Blm } \\
\text { Bli } \\
\text { IBlm } \\
\text { IBli }\end{array}$ & $\begin{array}{l}2 \\
2 \\
2 \\
2\end{array}$ & $\begin{array}{l}21,7 \\
24,2 \\
25,1 \\
26,1\end{array}$ & & & $\begin{array}{l}3,0 \\
2,7\end{array}$ \\
\hline
\end{tabular}

(1) Le pF est déterminé à partir des relations de la figure 4. 


\section{CONCLUSION GÉNÉRALE ET CONSÉQUENCES}

Dans la gamme des $\mathrm{pF}$ que l'on enregistre en fin de période pluvieuse (l à 3 ), en situation d'équilibre hydrique entre unités morphologiques à éléments $\Delta$ et à éléments $\Gamma$, les premières ont de plus faibles teneurs pondérales en eau. Mais, juste après l'infiltration d'une pluie, du fait d'une circulation non uniforme, les éléments structuraux les plus compacts restent à des $\mathrm{pF}$ plus élevés que des éléments plus poreux de même cote. Ce dernier phénomène accentue l'écart des teneurs en eau dû au premier.

Ainsi, plusieurs raisons expliquent qu'en conditions naturelles, après pluies, les zones les plus poreuses soient les plus humides. Nous tirons de ces résultats les 2 remarques suivantes:

1) Notre étude souligne la pertinence de la méthode de caractérisation morphologique de l'état structural préconisée par MANICHON (1982a) pour comprendre le comportement hydrique de la couche travaillée. Elle tient au fait de décrire la variabilité spatiale de la structure en distinguant plusieurs niveaux d'organisation dont chacun intervient sur le comportement hydrique du sol.
2) Les résultats renforcent la nécessité de tenir compte de l'état physique initial pour prévoir le comportement du sol sous l'effet des outils : les éléments structuraux les plus compacts, qui sont plus résistants aux actions mécaniques que des éléments plus poreux à teneur en eau identique, le deviennent plus encore de par leur comportement hydrique propre. La conduite des états physiques du sol par les façons culturales détermine donc les conditions de la conduite ultérieure. On aboutit ainsi à remettre en cause toute détermination de " jours disponibles " pour une opération qui ne tiendrait pas compte des états initiaux hérités du passé cultural, c'est-à-dire qui ne resituerait pas l'opération dans l'itinéraire technique (PAPY, 1982, 1986).

Reçu le 22 juillet 1985. Accepté le 27 février 1986.

\section{REMERCIEMENTS}

Je remercie sincèrement MM. M. Sebillotte et H. Manichon (Chaire d'Agronomie I.N.A.-P.G.), G, MonNIER (I.N.R.A., Science du Sol Avignon), D. Tessier (I.N.R.A., Science du Sol Versailles) pour l'aide qu'ils m'ont fournie dans la conduite de cette étude.

\section{RÉFÉRENCES BIBLIOGRAPHIQUES}

Chang R. K., 1968. Component potentials and hysteresis in water retention by compacted clay soil aggregates. Soil Sci., 105, 172-176.

Croney D., Coleman J. D., 1954. Soil structure in relation to soil suction (pF). J. Soil Sci., 5, 75-84.

Feodoroff A., 1965. Etude expérimentale de l'infiltration de l'eau non saturante. Thèse Paris, 90 p. (Public. I.N.R.A.).

Grimaldi M., 1981. Contribution à l'étude du tassement des sols : Evolution de la structure d'un matériau limoneux soumis à des contraintes mécaniques et hydriques. Thèse Rennes, $221 \mathrm{p}$.

Hénin S., Gras R., Monnier G., 1969. Le profil cultural. Masson, Paris, $332 \mathrm{p}$.

Klute A., 1982. Tillage effects on the hydraulic properties of soil : a review, 29-43. In : "Predicting tillage. Effects on soil physical properties and process », SSA special pub. 44, Madison, U.S.A., $198 \mathrm{p}$.

Manichon H., 1982a. Influence des systèmes de culture sur le profil cultural: élaboration d'une méthode de diagnostic basée sur l'observation morphologique. Thèse I.N.A.-P.G., Paris, 214 p. + annexes.

Manichon H., 1982b. L'action des outils sur le sol : Appréciation de leurs effets par la méthode du profil cutural. Sci. Sol, 3, 203-219.

Manichon H., Sebillotte M., 1975. Analyse et prévision des conséquences des passages successifs d'outils sur le profil cultural. Bull. Tech. Inf. Min. Agric., 302-303, 569-577.
Monnier G., Stengel P., Fies J. C., 1973. Une méthode de mesure de la densité apparente des petits agglomérats terreux. Application à l'analyse des systèmes de porosité du sol. Ann. Agron., 24 (5), 533545.

Papy F., 1982. Praticabilité du milieu et itinéraires techniques. Bull. Tech. Inf. Min. Agric., 370-372, 375-381.

Papy F., 1984. Comportement du sol sous l'action des façons de reprise d'un labour au printemps. Effets des conditions climatiques et de l'état structural. Thèse I.N.A.-P.G., Paris, 232 p. + annexes.

Papy F., 1986. La conduite des états physiques d'un sol cultivé. Analyse des effets de l'état initial (Bull. tech. Inf. Min. Agric., $n^{\circ}$ spécial "Organisation du travail ", à paraître).

Stakman V. P., Walk G. A., Van der Harst O. G., 1969. Détermination of soil moisture retension curves (I). Publ. of Inst. for Land and Water management Res., Wageningen (Pays-Bas), 19 p.

Tessier D., Berrier J., 1979. Utilisation de la microscopie à balayage dans l'étude des sols. Observation des sols humides soumis à différents pF. Sci. Sol, 1, 67-82.

Warkentin B., 1971. Effects of compaction on content and transmission of water soil. 126-153. In : "Compaction of Agricultural soils ". Am. Soc. of Agric. Eng., 471 p. 